\title{
A search for
}

\section{Dirac magnetic poles}

\author{
by L. HOFFMANN, W. O. LOCK, and G. VANDERHAEGHE \\ Nuclear Physics Division
}

\begin{abstract}
About 30 years ago Dirac predicted the existence of positive electrons as a natural consequence of his quantized theory of the electromagnetic field. As is well known, his prediction was experimentally verified rather quickly. At about the same time, Dirac showed that one can construct a theory which contains, as sources of the electromagnetic field, point magnetic poles (monopoles) besides point electric charges. The theory in fact is not at all complete; however, the existence of magnetic poles does not contradict any well-established law of nature. Therefore, many authors have proposed experiments in order to search for poles, either in cosmic radiation or among the secondary particles produced in high-energy collisions. For example, Bradner and Isbell, in 1958, carried out a series of such experiments at the Berkeley Bevatron. The fact that none of these experiments showed any evidence for the existence of poles may be interpreted as the consequence of either of two things:
\end{abstract}

1. Their mass was too large to allow their production (in pairs) at the energies reached with the accelerators used. (The energy of the Bevatron is enough to produce a proton-antiproton pair; the mass of the poles should then be larger than that of the proton.)

2. The cross-section for their production was so small that it was very unlikely they would be found amongst secondary cosmic rays.

The higher energies available at CERN make it possible to extend the search up to mass values for the poles nearly three times the proton mass. It was therefore suggested independently by Amaldi (November 1959) and Bradner (January 1960) that a series of experiments should be carried out at CERN to search for poles, using nuclear emulsions as detectors.

Eventually a mixed research group was set up for this work consisting of E. Amaldi, G. Baroni, H. G. de Carvalho and A. Manfredini, from the Institute of Physics of Rome, H. Bradner, from the Lawrence Radiation Laboratory at Berkeley, and L. Hoffmann and G. Vanderhaeghe, from CERN. Two types of experiment were carried out in 1961, and both gave a negative result.

It should be mentioned that similar experiments, but using counter techniques to detect the poles, were carried out in 1961 by a group at Brookhaven and by Mr. Fidecaro, G. Finocchiaro and G. Giacomelli at CERN; both groups also obtained negative results.
A third experiment using emulsions has been started at CERN in February of this year. It is more straightforward than the two previous ones and should give the most reliable upper limit of the cross-section for the production of poles in proton-nucleon collisions at $28 \mathrm{GeV}$. We will describe it here; the experimental apparatus used is shown in the accompanying photograph.

It is assumed that pairs of poles will be produced in a target, placed in the proton-synchrotron accelerator,

P. A.M. Dirac is an English physicist who introduced the relativistic quantum theory of the electron that is the basis of present-day quantum electrodynamics. He was a joint recipient of the Nobel Prize for Physics in 1933.

Mass and energy are interchangeable, so that if any fundamental particle is accelerated and given a sulficiently high energy the possibility exists of converting a large part of the combined mass and kinetic energy of the particle into the mass of some new, heavier particle. Conversely, before there can be any hope of producing any new particle postulated theoretically, a certain minimum energy is required, corresponding to its mass.

The gauss is the unit in which the strength of magnetic fields is measured. For example the earth's magnetic field is equivalent to about $1 / 5$ gauss while a small horseshoe magnet gives a field of a few hundred gauss.

Nuclear emulsions are special photographic emulsions, basically similar to the coating on an ordinary film. A charged nuclear particle passing through the emulsion causes ionization along its path, and this shows up as a trail of tiny black blobs on development of the emulsion.

A relativistic particle is one that is moving at such a high speed that the classical laws of mechanics are no longer completely accurate. For example any further gain in energy fends to increase its mass rather than its velocity, and 'relativistic' mechanics have to be used in any calculations.

Cross-section of a nuclear reaction is a measure of its probability of occurrence. For a common nuclear reaction the cross-section is about $10^{-24} \mathrm{~cm}^{2}$, so that a cross-section of $10^{-39} \mathrm{~cm}^{2}$ represents a reaction some $10^{15}$ times less probable. 
when it is bombarded by $28-\mathrm{GeV}$ protons. At a short distance downstream from the target, the poles enter a high magnetic field (about 20000 gauss) produced by a pair of pulsed coils (Krienen coils, partly visible at the bottom of the photograph to the right of the warning notice). The poles are deflected by this field in such a manner that a reasonable fraction of them are extracted from the vacuum chamber of the machine and 'sucked' through the upper coil. They are then steered by a smaller magnetic field (about 2000 gauss) inside a series of three solenoids, in which emulsion stacks are placed at different levels. (The solenoids are visible on the photograph, making an angle of $60^{\circ}$ to the horizontal). A shielding of lead protects the emulsion against direct radiation from the target.

Now it follows from Dirac's theory that poles lose energy very rapidly by ionization when passing through matter (relativistic poles ionize at least about 4700 times more than a relativistic elementary charged particle). Thus, in nuclear emulsion, a pole should leave a very distinctive heavy track. In the experiment we are describing, relatively insensitive emulsions were used, in conjunction with a special processing technique so that the general background from the synchrotron gives only a slight fogging after two hours of irradiation. The track of a pole should still show up clearly, however.

The emulsions have already been processed and are now being examined. If no poles are found, the experiment will allow an upper limit of the order of $10^{-39} \mathrm{~cm}^{2}$ to be assigned to the cross-section for the production of poles in proton-nucleon collisions at $28 \mathrm{GeV}$ (or 7,5 $\mathrm{GeV}$ in the centre-of-mass system)

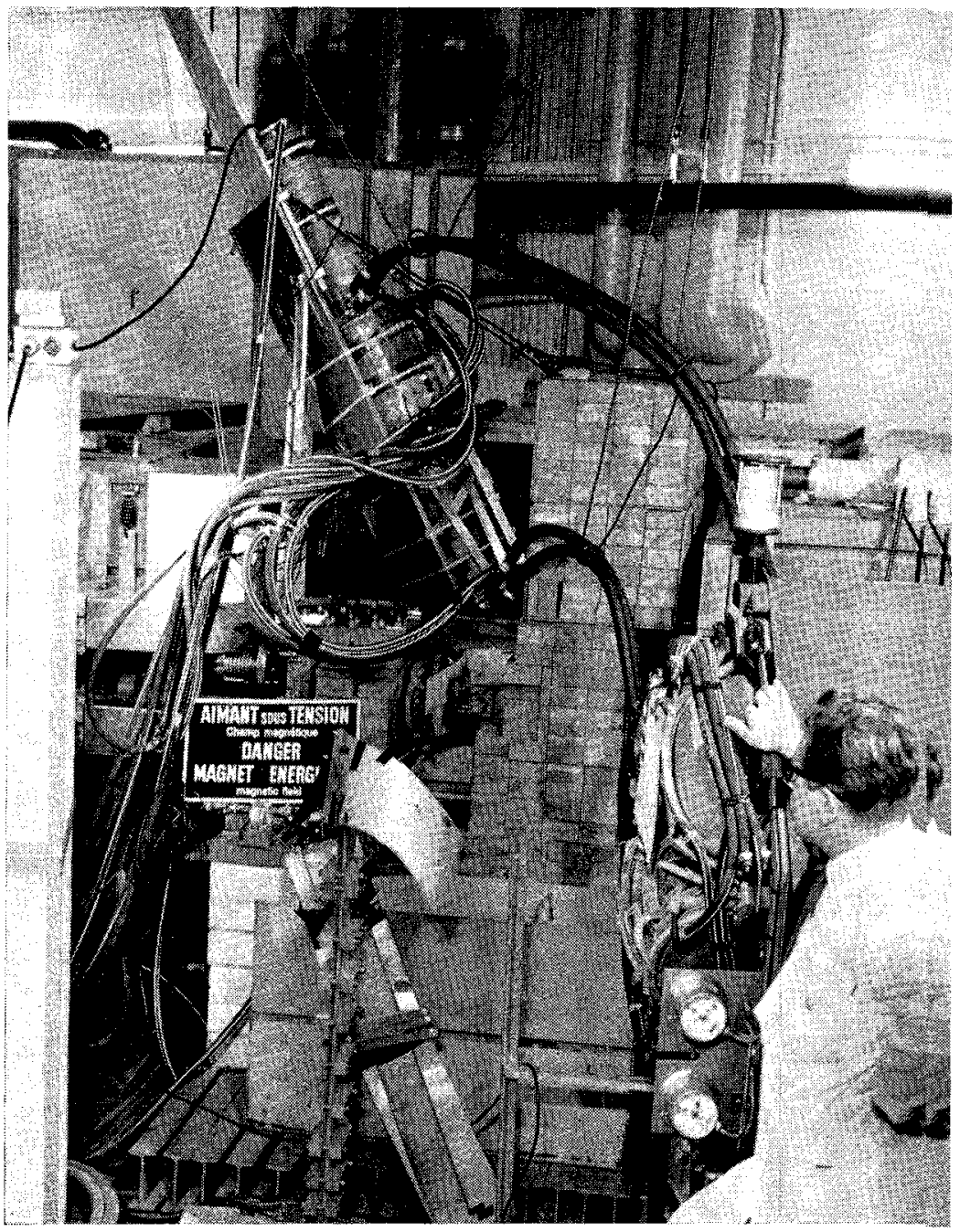

The experimental arrangement used in the search for magnetic monopoles. Immediately to the right of the warning notice can be seen the bus-bar connexions to the pulsed coil, and above these the thres solenoids that contain the nuclear emulsions slope up to the left.

\section{News from Alroad}

\section{ACCELERATOR DEVELOPMENT IN THE U.S.A.}

A simultaneous announcement on 1 February from the U.S. Afomic Energy Commission and the University of California gave the news that the 88-inch cyclotron at the University's Lawrence Radiation Laboratory had accelerated its first beam.

This machine, the latest in the battery of particle accelerators at the Laboratory devoted to exploring the atomic nucleus, is especially interesting, because of its 'spiral-ridge' design. Its general appearance is similar to that of other cyclotrons, including the $600-\mathrm{MeV}$ synchrocyclotron af CERN, but there is an important difference in the magnet. The fwo pole faces, $2235 \mathrm{~mm}$ ( 88 inches) in diameter, are not flat, but each have three spiral ridges, plateaus about $5 \mathrm{~mm}$ high, radiating rather like the arms of a starfish from the centre. In this way the strength of the magnetic field which acts on the accelerated parficles between the pole faces varies along the path of the beam, keeping it focused and 'in step' with the accelerating frequency to a higher energy than is possible with a simple cyclotron.

Protons will be accelerated to $50 \mathrm{MeV}$, deuterons (nuclei of heavy hydrogen) to $60 \mathrm{MeV}$, and alpha particles (nuclei of helium) to $120 \mathrm{MeV}$. Later the machine will be used to accelerate heavier nuclei, such as carbon-12 or oxygen-16, to about $10 \mathrm{MeV}$. Although these energies are not high compared to those produced by many larger machines, the beam intensity is expected to be some thousand million million (1015) particles per second (about 200 times higher than that of the CERN synchro-cyclotron). The energy can also be varied over a very wide range by adjusting the accelerating frequency.

The new accelerator will allow more accurate measurement of phenomena occuring in nuclear scattering experiments and will permit the production in larger quantities than before of selected isotopes of all the elements, including the man-made elements heavier than uranium. New areas are expected to be opened up in atomic-beam research, nuclear spectroscopy, and the biological effects of radiation.

Another giant accelerator went into operation in the U.S.A. in March. This was the Cambridge Eleciron Accelerafor, a synchrotron for accelerafing electrons to $6 \mathrm{GeV}$. Similar in principle to the CERN proton synchrotron, it is 72

(continued on p. 12) 